\title{
Dark Matter Search in Missing Energy Events with NA64
}

D. Banerjee, ${ }^{4,5}$ V. E. Burtsev, ${ }^{2}$ A. G. Chumakov, ${ }^{13}$ D. Cooke, ${ }^{6}$ P. Crivelli, ${ }^{15}$ E. Depero, ${ }^{15}$ A. V. Dermenev, ${ }^{7}$ S. V. Donskov, ${ }^{11}$ R. R. Dusaev, ${ }^{13}$ T. Enik, ${ }^{2}$ N. Charitonidis, ${ }^{4}$ A. Feshchenko, ${ }^{2}$ V. N. Frolov, ${ }^{2}$ A. Gardikiotis, ${ }^{10}$ S. G. Gerassimov, ${ }^{8,3}$ S. N. Gninenko $\odot,{ }^{7, *}$ M. Hösgen, ${ }^{1}$ M. Jeckel, ${ }^{4}$ A. E. Karneyeu, ${ }^{7}$ G. Kekelidze, ${ }^{2}$ B. Ketzer, ${ }^{1}$ D. V. Kirpichnikov, ${ }^{7}$ M. M. Kirsanov, ${ }^{7}$ I. V. Konorov, ${ }^{8,3}$ S. G. Kovalenko, ${ }^{14}$ V. A. Kramarenko, ${ }^{2,9}$ L. V. Kravchuk, ${ }^{7}$ N. V. Krasnikov, ${ }^{2,7}$ S. V. Kuleshov, ${ }^{12}$ V. E. Lyubovitskij, ${ }^{13,14}$ V. Lysan, ${ }^{2}$ V. A. Matveev, ${ }^{2,7}$ Yu. V. Mikhailov, ${ }^{11}$ L. Molina Bueno, ${ }^{15}$ D. V. Peshekhonov, ${ }^{2}$ V. A. Polyakov, ${ }^{11}$ B. Radics, ${ }^{15}$ R. Rojas, ${ }^{14}$ A. Rubbia, ${ }^{15}$ V. D. Samoylenko, ${ }^{11}$ D. Shchukin, ${ }^{8}$ V. O. Tikhomirov, ${ }^{8}$ I. Tlisova, ${ }^{7}$ D. A. Tlisov, ${ }^{7}$ A. N. Toropin, ${ }^{7}$ A. Yu. Trifonov, ${ }^{13}$ B. I. Vasilishin, ${ }^{13}$ G. Vasquez Arenas, ${ }^{14}$ P. V. Volkov, ${ }^{2,9}$ V. Yu. Volkov, ${ }^{9}$ and P. Ulloa ${ }^{14}$

(NA64 Collaboration)

\author{
${ }^{1}$ Universität Bonn, Helmholtz-Institut für Strahlen-und Kernphysik, 53115 Bonn, Germany \\ ${ }^{2}$ Joint Institute for Nuclear Research, 141980 Dubna, Russia \\ ${ }^{3}$ Technische Universität München, Physik Department, 85748 Garching, Germany \\ ${ }^{4}$ CERN, European Organization for Nuclear Research, CH-1211 Geneva, Switzerland \\ ${ }^{5}$ University of Illinois at Urbana Champaign, Urbana, 61801-3080 Illinois, USA \\ ${ }^{6}$ Department of Physics and Astronomy, University College London, Gower St., London WC1E 6BT, United Kingdom \\ ${ }^{7}$ Institute for Nuclear Research, 117312 Moscow, Russia \\ ${ }^{8}$ P.N. Lebedev Physical Institute, Moscow, Russia, 119991 Moscow, Russia \\ ${ }^{9}$ Skobeltsyn Institute of Nuclear Physics, Lomonosov Moscow State University, 119991 Moscow, Russia \\ ${ }^{10}$ Physics Department, University of Patras, 26504 Patras, Greece \\ ${ }^{11}$ State Scientific Center of the Russian Federation Institute for High Energy Physics of National Research Center 'Kurchatov Institute' \\ (IHEP), 142281 Protvino, Russia \\ ${ }^{12}$ Departamento de Ciencias Fiisicas, Universidad Andres Bello, Sazié 2212, Piso 7, Santiago, Chile \\ ${ }^{13}$ Tomsk State Pedagogical University, 634061 Tomsk, Russia \\ ${ }^{14}$ Universidad Técnica Federico Santa María, 2390123 Valparaíso, Chile \\ ${ }^{15}$ ETH Zürich, Institute for Particle Physics and Astrophysics, CH-8093 Zürich, Switzerland
}

(Received 5 June 2019; published 18 September 2019)

\begin{abstract}
A search for sub-GeV dark matter production mediated by a new vector boson $A^{\prime}$, called a dark photon, is performed by the NA64 experiment in missing energy events from $100 \mathrm{GeV}$ electron interactions in an active beam dump at the CERN SPS. From the analysis of the data collected in the years 2016, 2017, and 2018 with $2.84 \times 10^{11}$ electrons on target no evidence of such a process has been found. The most stringent constraints on the $A^{\prime}$ mixing strength with photons and the parameter space for the scalar and fermionic dark matter in the mass range $\lesssim 0.2 \mathrm{GeV}$ are derived, thus demonstrating the power of the active beam dump approach for the dark matter search.
\end{abstract}

DOI: 10.1103/PhysRevLett.123.121801

The idea that in addition to gravity a new force between the dark and visible matter transmitted by a vector boson, $A^{\prime}$, called dark photon, might exist is quite exciting [1-4]. The $A^{\prime}$ can have a mass in the sub-GeV mass range, and couple to the standard model (SM) via kinetic mixing with

Published by the American Physical Society under the terms of the Creative Commons Attribution 4.0 International license. Further distribution of this work must maintain attribution to the author(s) and the published article's title, journal citation, and DOI. Funded by SCOAP ${ }^{3}$. the ordinary photon, described by the term $(\epsilon / 2) F_{\mu \nu}^{\prime} F^{\mu \nu}$ and parametrized by the mixing strength $\epsilon$. An example of the Lagrangian of the SM extended by the dark sector (DS) is given by

$$
\begin{aligned}
\mathcal{L}= & \mathcal{L}_{\mathrm{SM}}-\frac{1}{4} F_{\mu \nu}^{\prime} F^{\prime \mu \nu}+\frac{\epsilon}{2} F_{\mu \nu}^{\prime} F^{\mu \nu}+\frac{m_{A^{\prime}}^{2}}{2} A_{\mu}^{\prime} A^{\prime \mu} \\
& +i \bar{\chi} \gamma^{\mu} \partial_{\mu} \chi-m_{\chi} \bar{\chi} \chi-e_{D} \bar{\chi} \gamma^{\mu} A_{\mu}^{\prime} \chi
\end{aligned}
$$

where the massive $A_{\mu}^{\prime}$ field is associated with the spontaneously broken $U_{D}(1)$ gauge group, $F_{\mu \nu}^{\prime}=\partial_{\mu} A_{\nu}^{\prime}-\partial_{\nu} A_{\mu}^{\prime}$, and $m_{A^{\prime}}, m_{\chi}$ are, respectively, the masses of the $A^{\prime}$ and dark 
matter (DM) particles, $\chi$, which are treated as Dirac fermions coupled to $A_{\mu}^{\prime}$ with the dark coupling strength $e_{D}$ of the $U(1)_{D}$ gauge interactions. The mixing term of (1) results in the interaction $\mathcal{L}_{\text {int }}=\epsilon e A_{\mu}^{\prime} J_{e m}^{\mu}$ of dark photons with the electromagnetic current $J_{e m}^{\mu}$ with a strength $\epsilon e$, where $e$ is the electromagnetic coupling and $\epsilon \ll 1$ [5-7]. Such small values of $\epsilon$ can be obtained in grand unified theories from loop effects of particles charged under both the dark $U_{D}(1)$ and SM $U(1)$ interactions with a typical one-loop value $\epsilon=e e_{D} / 16 \pi^{2} \simeq 10^{-2}-10^{-4}$ [7], or from two-loop contributions resulting in $\epsilon \simeq 10^{-3}-10^{-5}$. The accessibility of these values at accelerator experiments has motivated a worldwide effort towards dark forces and other portals between the visible and dark sectors; see Refs. [4,8-17] for a review.

If the $A^{\prime}$ is the lightest state in the dark sector, then it would decay mainly visibly to SM leptons $l$ (or hadrons); see, e.g., [18-23], and also [17]. In the presence of light DM states $\chi$ with the masses $m_{\chi}<m_{A^{\prime}} / 2$, the $A^{\prime}$ would predominantly decay invisibly into those particles provided that $e_{D}>\epsilon e$. Various dark sector models motivate the existence of sub-GeV scalar and Majorana or pseudo-Dirac DM coupled to the $A^{\prime}[13,14,17,24-28]$. To interpret the observed abundance of DM relic density, the requirement of the thermal freeze-out of DM annihilation into visible matter through $\gamma-A^{\prime}$ mixing allows one to derive a relation

$$
\alpha_{D} \simeq 0.02 f\left(\frac{10^{-3}}{\epsilon}\right)^{2}\left(\frac{m_{A^{\prime}}}{100 \mathrm{MeV}}\right)^{4}\left(\frac{10 \mathrm{MeV}}{m_{\chi}}\right)^{2},
$$

where $\alpha_{D}=e_{D}^{2} / 4 \pi$ and the parameter $f$ depends on $m_{A^{\prime}}$ and $m_{\chi}$ [29]. For $m_{A^{\prime}} / m_{\chi}=3, f \lesssim 10$ for a scalar [24], and $f \lesssim 1$ for a fermion [25]. This prediction provides an important target for the $\left(\epsilon, m_{A^{\prime}}\right)$ parameter space, which can be probed at the CERN SPS energies. Models introducing the invisible $A^{\prime}$ also may explain various astrophysical anomalies [30] and are subject to various experimental constraints leaving, however, a large area that is still unexplored [24,31-40].

In this work we report new results on the search for the $A^{\prime}$ mediator and light dark matter (LDM) in the fixed-target experiment NA64 at the CERN SPS. In the following we assume that the $A^{\prime}$ invisible decay mode is predominant, i.e., $\Gamma\left(A^{\prime} \rightarrow \bar{\chi} \chi\right) / \Gamma_{\text {tot }} \simeq 1$. If such invisible $A^{\prime}$ exists, many crucial questions about its coupling constants, mass scale, decay modes, etc. arise. One possible way to answer these questions is to search for the $A^{\prime}$ in fixed-target experiments. The $A^{\prime}$ s could be produced by a high-intensity beam in a dump and generate a flux of DM particles through the $A^{\prime} \rightarrow \bar{\chi} \chi$ decay, which can be detected through the scattering off electrons in the far target $[24,25,31,34,41,42]$. The signal event rate in the detector in this case scales as $\epsilon^{2} y \propto \epsilon^{4} \alpha_{D}$, with one $\epsilon^{2}$ associated with the $A^{\prime}$ production in the dump and $\epsilon^{2} \alpha_{D}$ coming from the $\chi$ particle scattering in the detector, and with the parameter $y$ defined as $y=\epsilon^{2} \alpha_{D}\left(m_{\chi} / m_{A^{\prime}}\right)^{4}$. Another method, discussed in this work and proposed in Refs. [43,44], is based on the detection of the missing energy, carried away by the hard bremsstrahlung $A^{\prime}$ produced in the process $e^{-} Z \rightarrow e^{-} Z A^{\prime}$; $A^{\prime} \rightarrow$ invisible of high-energy electrons scattering in the active beam dump target. The advantage of this type of experiment compared to the beam dump ones is that its sensitivity is proportional to $\epsilon^{2}$, associated with the $A^{\prime}$ production and its subsequent prompt invisible decay.

The NA64 detector is schematically shown in Fig. 1. The experiment employed the optimized $\mathrm{H} 4100 \mathrm{GeV}$ electron beam [45]. The beam has a maximal intensity $\simeq 10^{7}$ electrons per SPS spill of $4.8 \mathrm{~s}$ produced by the primary $400 \mathrm{GeV}$ proton beam with an intensity of few $10^{12}$ protons on target. The detector utilized the beam defining scintillator (Sc) counters $S_{1-4}$ and veto $V_{1,2}$, a magnetic spectrometer consisting of two successive dipole magnets $\mathrm{MBPL}_{1,2}$ with the integral magnetic field of $\simeq 7 \mathrm{Tm}$ and a low-material-budget tracker. The tracker was a set of two upstream Micromegas chambers $\mathrm{MM}_{1,2}$, and four $\mathrm{MM}_{3-6}$, downstream stations, as well as two straw-tube $\mathrm{ST}_{1,2}$ and $\mathrm{GEM}_{1,2}$ chambers allowing the measurements of $e^{-}$ momenta with the precision $\delta p / p \simeq 1 \%$ [46]. To enhance electron identification, synchrotron radiation (SR) emitted in the MBPL magnetic field was used for their efficient tagging with a SR detector (SRD), which was an array of a $\mathrm{PbSc}$ sandwich calorimeter of a very fine segmentation $[43,47]$. By using the SRD the initial admixture of the hadron contamination in the beam $\pi / e^{-} \lesssim 10^{-2}$ was further suppressed by a factor $\simeq 10^{3}$. The detector was also equipped with an active dump target, which is an electromagnetic calorimeter (ECAL), a matrix of $6 \times 6$ Shashliktype modules assembled from $\mathrm{Pb}$ and $\mathrm{Sc}$ plates for measurement of the electron energy $E_{\mathrm{ECAL}}$. Each module has $\simeq 40$ radiation lengths $\left(X_{0}\right)$ with the first $4 X_{0}$ serving as a preshower detector. Downstream of the ECAL, the detector was equipped with a large high-efficiency veto counter VETO, and a massive, hermetic hadronic calorimeter (HCAL) of $\simeq 30$ nuclear interaction lengths in total. The modules $\mathrm{HCAL}_{1-3}$ provided an efficient veto to detect muons or hadronic secondaries produced in the $e^{-} A$ interactions in the target. The events were collected with the hardware trigger requiring an in-time cluster in the ECAL with the energy $E_{\mathrm{ECAL}} \lesssim 80 \mathrm{GeV}$. The search described in this paper uses the data samples of $n_{\mathrm{EOT}}=0.43 \times 10^{11}, 0.56 \times 10^{11}$ and $1.85 \times 10^{11}$ electrons on target (EOT), collected in the years 2016, 2017, and 2018 with the beam intensities in the range $\simeq(1.4-6) \times 10^{6}$, $\simeq(5-6) \times 10^{6}$, and $\simeq(5-9) \times 10^{6} e^{-}$per spill, respectively. Data corresponding it total to $2.84 \times 10^{11}$ EOT from these three runs (hereafter called respectively runs I, II, and III) were processed with selection criteria similar to the one used in Ref. [38] and finally combined as described below. 


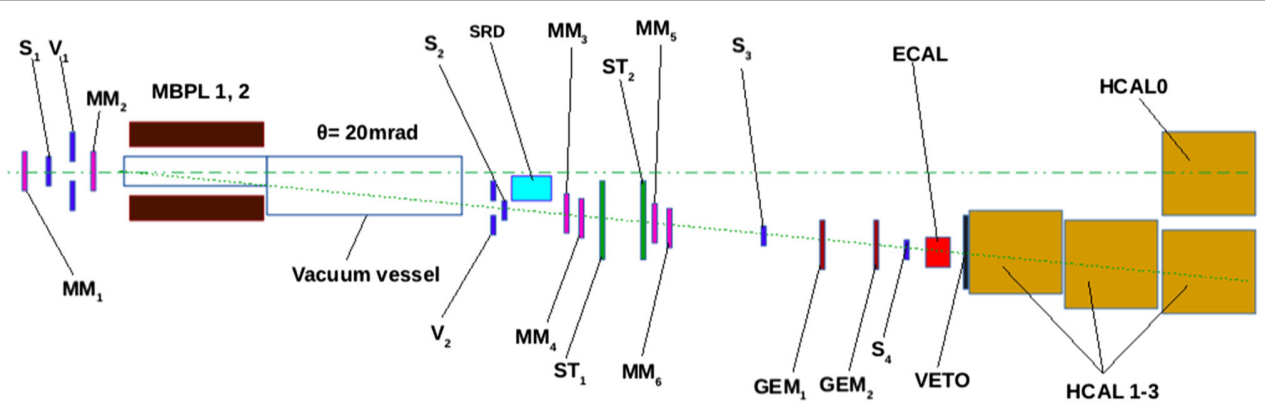

FIG. 1. Schematic illustration of the setup to search for $A^{\prime} \rightarrow$ invisible decays of the bremsstrahlung $A^{\prime}$ s produced in the reaction $e Z \rightarrow e Z A^{\prime}$ of $100 \mathrm{GeV} e^{-}$incident on the active ECAL target.

Compared to the analysis of Ref. [38], a number of improvements, in particular, in the track reconstruction were made in the 2018 run to increase the overall efficiency. Also, the zero-degree calorimeter $\mathrm{HCAL}_{0}$ was used to reject events accompanied by hard neutrals from the upstream $e^{-}$interactions; see Fig. 1.

In order to avoid biases in the determination of selection criteria for signal events, a blind analysis was performed. Candidate events were requested to have the missing energy $E_{\text {miss }}=E_{0}-E_{\mathrm{ECAL}}>50 \mathrm{GeV}$. The signal box $\left(E_{\mathrm{ECAL}}<50 \mathrm{GeV} ; E_{\mathrm{HCAL}}<1 \mathrm{GeV}\right)$ was defined based on the energy spectrum calculations for $A^{\prime}$ s emitted by $e^{ \pm}$ from the electromagnetic $(e-m)$ shower generated by the primary $e^{-} \mathrm{s}$ in the target $[48,49]$. A Geant4 [50,51] based Monte Carlo (MC) simulation used to study the detector performance, signal acceptance, and background level, as well as the analysis procedure including selection of cuts and estimate of the sensitivity are described in detail in Ref. [38].

The left panel in Fig. 2 shows the distribution of $\simeq 3 \times 10^{4}$ events from the reaction $e^{-} Z \rightarrow$ anything in the $\left(E_{\mathrm{ECAL}} ; E_{\mathrm{HCAL}}\right)$ plane measured with loose selection criteria requiring mainly the presence of a beam $e^{-}$ identified with the SR tag. Events from area I originate from the QED dimuon production, dominated by the reaction $e^{-} Z \rightarrow e^{-} Z \gamma ; \gamma \rightarrow \mu^{+} \mu^{-}$with a hard bremsstrahlung photon conversion on a target nucleus and characterized by the energy of $\simeq 10 \mathrm{GeV}$ deposited by the dimuon pair in the HCAL. This rare process was used as a benchmark allowing us to verify the reliability of the MC simulation, correct the signal acceptance, cross-check systematic uncertainties, and background estimate [38]. Region II shows the SM events from the hadron electroproduction in the target that satisfy the energy conservation $E_{\mathrm{ECAL}}+E_{\mathrm{HCAL}} \simeq 100 \mathrm{GeV}$ within the energy resolution of the detectors.

Finally, the following selection criteria were chosen to maximize the acceptance for signal events and to minimize background. (i) The incoming particle track should have the momentum $100 \pm 3 \mathrm{GeV}$ and a small angle with respect to the beam axis to reject large angle tracks from the upstream $e^{-}$interactions. (ii) The energy deposited in the SRD detector should be within the SR range emitted by $e^{-} \mathrm{s}$ and in time with the trigger. (iii) The lateral and
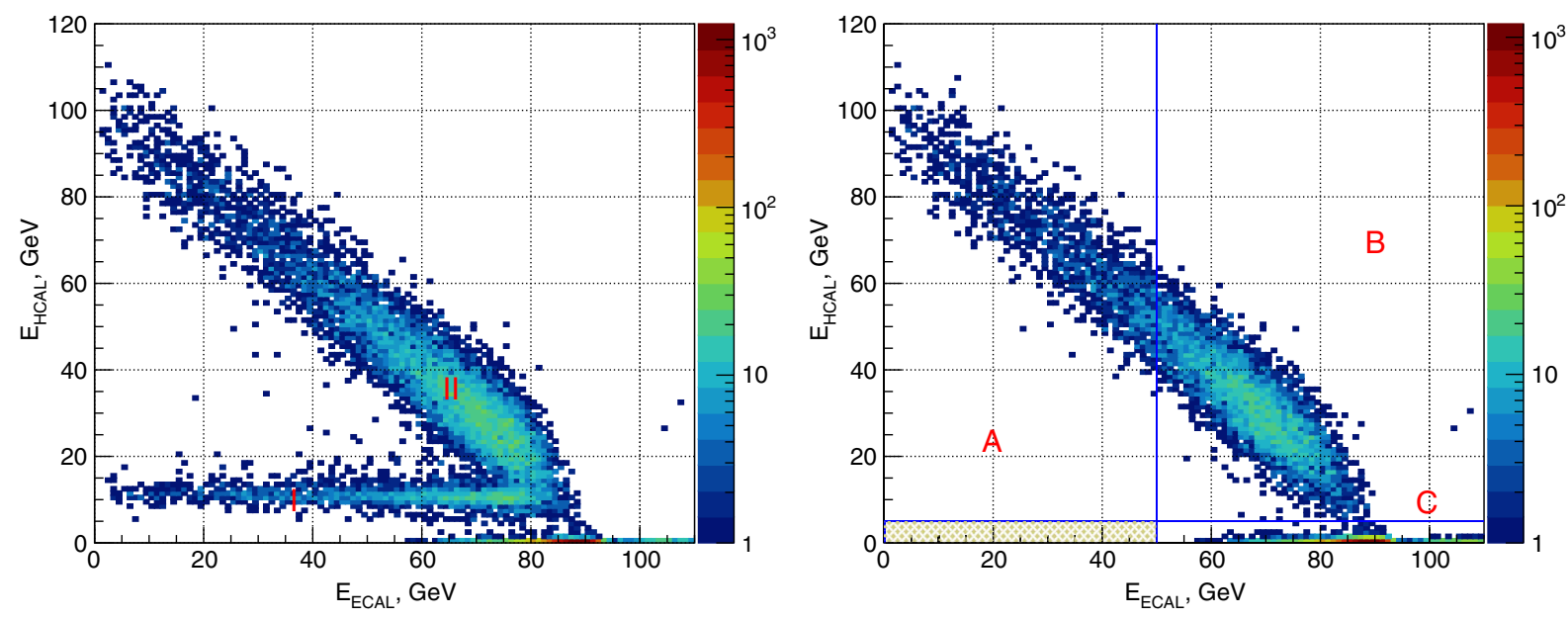

FIG. 2. The left panel shows the measured distribution of events in the $\left(E_{\mathrm{ECAL}} ; E_{\mathrm{HCAL}}\right)$ plane from the combined run data at the earlier phase of the analysis. The right panel shows the same distribution after applying all selection criteria. The shaded area is the signal box, which contains no events. The size of the signal box along the $E_{\mathrm{HCAL}}$ axis is increased by a factor of 5 for illustration purposes. The side bands $A$ and $C$ are the ones used for the background estimate inside the signal region. 
longitudinal shape of the shower in the ECAL should be consistent with the one expected for the signal shower [48]. (iv) There should be no multiple hits activity in the strawtube chambers, which was an effective cut against hadron electroproduction in the beam material upstream of the dump, and no activity in VETO. Only $\simeq 1.6 \times 10^{4}$ events passed these criteria from combined runs.

There are several background sources shown in Table I that may fake the signal: (i) loss of dimuons due to statistical fluctuations of the signal or muon decays, (ii) decays in flight of mistakenly SRD tagged $\pi, K$ (iii) the energy loss from the $e^{-}$hadronic interactions in the beam line due to the insufficient downstream detector coverage, and (iv) punch-through of leading neutral hadrons $\left(n, K_{L}^{0}\right)$ produced in the $e^{-}$interactions in the target. The backgrounds (i) and (ii) were simulated with the full statistics of the data. The background estimate in the case (iii) was mainly obtained from data by the extrapolation of events from the sideband $C\left(E_{\mathrm{ECAL}}>50 \mathrm{GeV} ; E_{\mathrm{HCAL}}<1 \mathrm{GeV}\right)$ shown in the right panel of Fig. 2 into the signal region and assessing the systematic errors by varying the fit functions selected as described in Ref. [38]. The shape of the extrapolation functions was taken from the analysis of a much larger data sample of events from case (iv), and crosschecked with simulations of the $e^{-}$hadronic interactions in the dump. For case (iv), events from the region $A$ $\left(E_{\mathrm{ECAL}}<50 \mathrm{GeV} ; E_{\mathrm{HCAL}}>1 \mathrm{GeV}\right)$ of Fig. 2, which are pure neutral hadronic secondaries produced in the ECAL, were used. The background (iv) was extracted from the data themselves by using the longitudinal segmentation of HCAL for the conservative punch-through probability estimate. After determining all the selection criteria and background levels, we unblind the data. No event in the signal box was found, as shown in Fig. 2, allowing us to obtain the $m_{A^{\prime}}$-dependent upper limits on the mixing strength.

In the final combined statistical analysis, runs I-III were analyzed simultaneously using the multibin limit setting technique [38] based on the RooStats package [52]. First, the background estimate, efficiencies, and their corrections and uncertainties were used to optimize the main cut defining the signal box, by comparing sensitivities, defined as an average expected limit calculated using the profile likelihood method. The calculations were done with

TABLE I. Expected background for $2.84 \times 10^{11}$ EOT.

\begin{tabular}{lc}
\hline \hline Background source & Background, $n_{b}$ \\
\hline (i) Dimuons & $0.024 \pm 0.007$ \\
(ii) $\pi, K \rightarrow e \nu, K_{e 3}$ decays & $0.02 \pm 0.01$ \\
(iii) $e^{-}$hadron interactions in the beam line & $0.43 \pm 0.16$ \\
(iv) $e^{-}$hadron interactions in the target & $<0.044$ \\
(v) Punch-through $\gamma$ 's, cracks, holes & $<0.01$ \\
Total $n_{b}$ (conservatively) & $0.53 \pm 0.17$ \\
\hline \hline
\end{tabular}

uncertainties used as nuisance parameters, assuming their log-normal distributions [53]. For this optimization, the most important inputs were the expected values from the background extrapolation into the signal region from the data samples of runs I-III with their errors estimated from the variation of the extrapolation functions. The optimal cut was found to be weakly dependent on the $A^{\prime}$ mass choice and can be safely set to $E_{\mathrm{ECAL}} \lesssim 50 \mathrm{GeV}$ for the whole mass range.

The combined 90\% confidence level (C.L.) upper limits for $\epsilon$ were determined by using the modified frequentist approach for confidence levels, taking the profile likelihood as a test statistic in the asymptotic approximation [54-56]. The total number of expected signal events in the signal box was the sum of expected events from the three runs,

$$
N_{A^{\prime}}=\sum_{i=1}^{3} N_{A^{\prime}}^{i}=\sum_{i=1}^{3} n_{\mathrm{EOT}}^{i} \epsilon_{A^{\prime}}^{i} n_{A^{\prime}}^{i}\left(\epsilon, m_{A^{\prime}}, \Delta E_{e}\right),
$$

where $\epsilon_{A^{\prime}}^{i}$ is the signal efficiency in run $i$, and $n_{A^{\prime}}^{i}\left(\epsilon, m_{A^{\prime}}, \Delta E_{A^{\prime}}\right)$ is the signal yield per EOT generated in the energy range $\Delta E_{e}$. Each $i$ th entry in this sum was calculated with simulations of signal events and processing them through the reconstruction program with the same selection criteria and efficiency corrections as for the data sample from run $i$. The combined $90 \%$ C.L. exclusion limits on the mixing strength as a function of the $A^{\prime}$ mass, calculated by taken into account the expected backgrounds and estimated systematic errors, can be seen in Fig. 3. The derived bounds are currently the best for the mass range $0.001 \lesssim m_{A^{\prime}} \lesssim 0.2 \mathrm{GeV}$ obtained from direct searches of $A^{\prime} \rightarrow$ invisible decays [17].

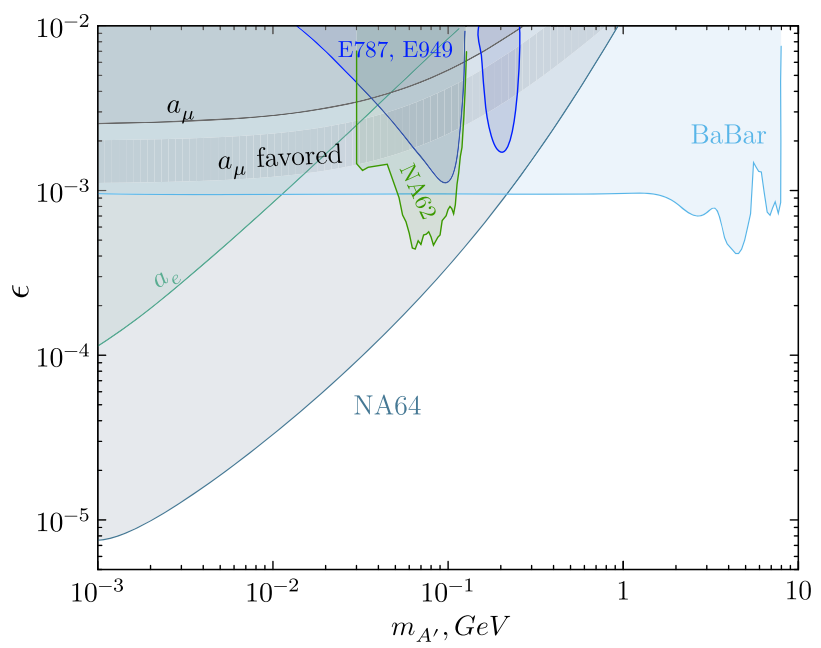

FIG. 3. The NA64 90\% C.L. exclusion region in the $\left(m_{A^{\prime}}, \epsilon\right)$ plane. Constraints from the E787 and E949 [32,33], BABAR [39], and recent NA62 [40] experiments, as well as the muon $\alpha_{\mu}$ favored area are also shown. For more limits from indirect searches and planned measurements see, e.g., Refs. [12-14]. 

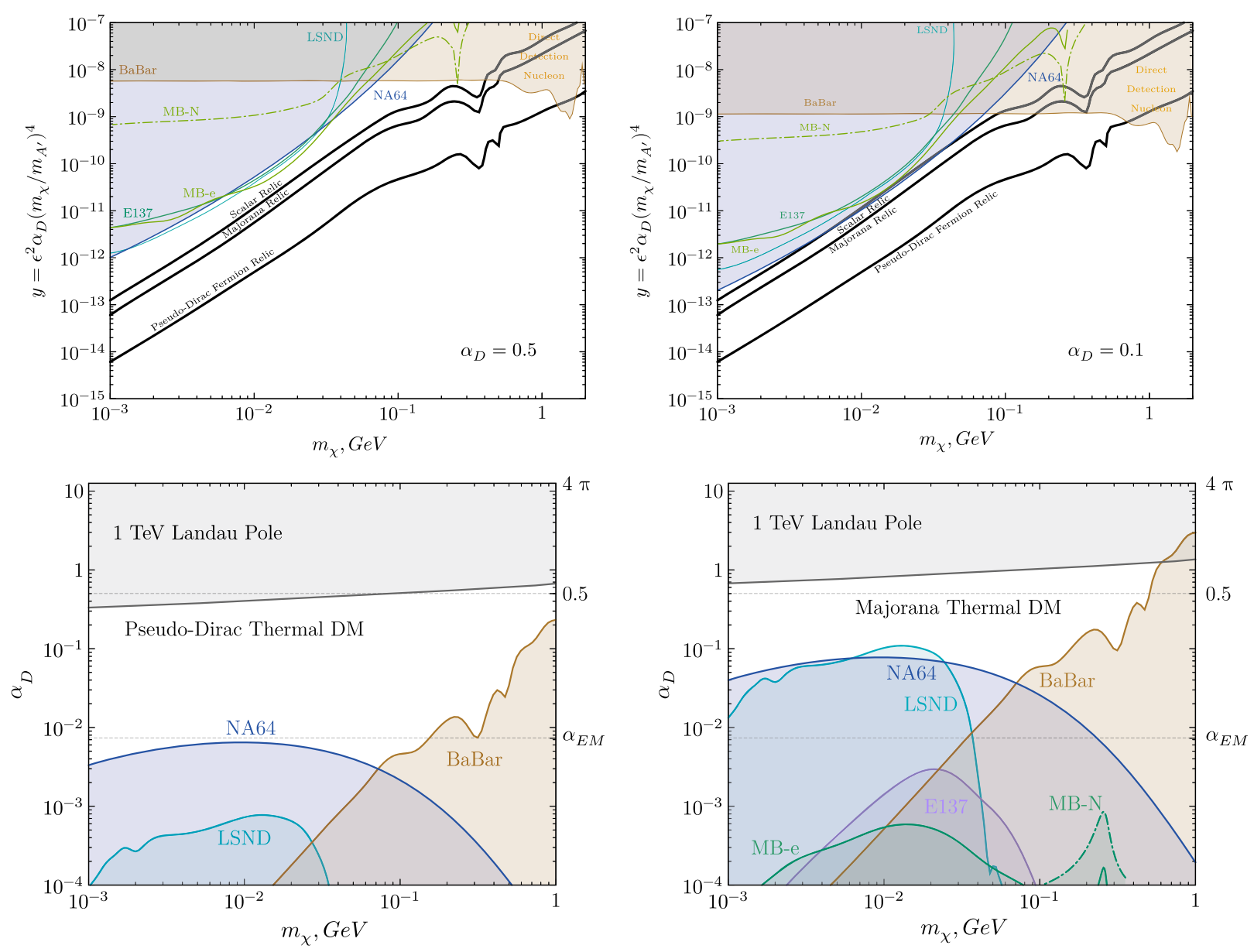

FIG. 4. The top row shows the NA64 limits in the (y; $m_{\gamma}$ ) plane obtained for $\alpha_{D}=0.5$ (left panel) and $\alpha_{D}=0.1$ (right panel) from the full 2016-2018 data set. The bottom row shows the NA64 constraints in the $\left(\alpha_{D} ; m_{\chi}\right)$ plane on the pseudo-Dirac (left panel) and Majorana (right panel) DM. The limits are shown in comparison with bounds obtained in Refs. [12,13,25-27] from the results of the LSND [24,34], E137 [35], MiniBooNE [37], BABAR [39], and direct detection [59] experiments. The favored parameters to account for the observed relic DM density for the scalar, pseudo-Dirac, and Majorana type of light DM are shown as the lowest solid line in top plots; see, e.g., [16].

The overall signal efficiency $\epsilon_{A^{\prime}}$ is slightly $m_{A^{\prime}}, E_{A^{\prime}}$ dependent and is given by the product of efficiencies accounting for the geometrical acceptance (0.97), the track $(\simeq 0.83)$, SRD ( $\gtrsim 0.95)$, VETO (0.94), and HCAL (0.94) signal reconstruction, and the DAQ dead time (0.93). The signal acceptance loss due to pileup was $\simeq 8 \%$ for highintensity runs. The VETO and HCAL efficiency was defined as a fraction of events below the corresponding zero-energy thresholds. The spectrum of the energy distributions in these detectors from the leak of the signal shower energy in the ECAL was simulated for different $A^{\prime}$ masses [48] and cross-checked with measurements at the $e^{-}$beam. The uncertainty in the VETO and HCAL efficiency for the signal events, dominated mostly by the pileup effect from penetrating hadrons in the high-intensity run III, was estimated to be $\lesssim 4 \%$. The trigger efficiency was found to be 0.95 with a small uncertainty $2 \%$. The $A^{\prime}$ acceptance was evaluated by taking into account the selection efficiency for the $e-m$ shower shape in the ECAL from signal events [48]. The $A^{\prime}$ production cross section in the primary reaction was obtained with the exact tree-level calculations as described in Ref. [49]. An additional uncertainty in the $A^{\prime}$ yield $\simeq 10 \%$ was conservatively accounted for the difference between the predicted and measured dimuon yield [36,38], which was the dominant source of systematic uncertainties on the expected number of signal events. The total signal efficiency $\epsilon_{A^{\prime}}$ for high(low-) intensity runs varied from $0.53 \pm 0.09(0.69 \pm 0.09)$ to $0.48 \pm 0.08(0.55 \pm 0.07)$, decreasing for the higher $A^{\prime}$ masses.

Using constraints on the cross section of the DM annihilation freeze-out [see Eq. (2)], and obtained limits on mixing strength, one can derive constraints on the LDM models, which are shown in the $\left(y ; m_{\chi}\right)$ and $\left(\alpha_{D} ; m_{\chi}\right)$ planes in Fig. 4 for masses $m_{\chi} \lesssim 1 \mathrm{GeV}$. On the same plot one can also see the favored $y$ parameter curves for scalar, 
pseudo-Dirac (with a small splitting) and Majorana scenario of LDM obtained by taking into account the observed relic DM density [16]. The limits on the variable $y$ are calculated under the convention $\alpha_{D}=0.1$ and 0.5 , and $m_{A^{\prime}}=3 m_{\chi}[13,14]$ and shown also for comparison with bounds from other experiments. This choice of the $\alpha_{D}$ region is compatible with the bounds derived based on the running of the dark gauge coupling arguments of Refs. [49,57]. It should be noted that for smaller values of $\alpha_{D}$ the NA64 limits will be stronger, due to the fact that the signal rate in our case scales as $\epsilon^{2}$, instead of $\epsilon^{4} \alpha_{D}$ as for beam dump searches. The bounds on $\alpha_{D}$ for the case of pseudo-Dirac fermions shown in Fig. 4 (left panel in the bottom row) were calculated by taking the value $f=0.25$, while for the Majorana case (right panel) the value $f=3$ in Eq. (2) [38] was used [58]. One can see that using the NA64 approach allows us to obtain more stringent bounds on $\epsilon, y$, $\alpha_{D}$ for the mass range $m_{\chi} \lesssim 0.1 \mathrm{GeV}$ than the limits obtained from the results of classical beam dump experiments, thus demonstrating its power for the dark matter search. Further improving of the sensitivity is expected after the NA64 detector upgrade.

We gratefully acknowledge the support of the CERN management and staff and the technical staffs of the participating institutions for their vital contributions. This work was supported by the Helmholtz-Institut für Strahlen-und Kernphysik (HISKP), University of Bonn (Germany), Joint Institute for Nuclear Research (JINR) (Dubna), the Ministry of Science and Higher Education (MSHE) and RAS (Russia), ETH Zurich and SNSF Grant No. 169133 (Switzerland), and FONDECYT Grants No. 1191103, No. 1190845, and No. 3170852, UTFSM PIM 18 13, and Basal Grant No. FB0821 CONICYT (Chile).

*Corresponding author.

Sergei.Gninenko@cern.ch

[1] P. Fayet, Phys. Lett. 95B, 285 (1980).

[2] M. Pospelov, A. Ritz, and M. B. Voloshin, Phys. Lett. B 662, 53 (2008).

[3] N. Arkani-Hamed, D. P. Finkbeiner, T. R. Slatyer, and N. Weiner, Phys. Rev. D 79, 015014 (2009).

[4] J. Jaeckel and A. Ringwald, Annu. Rev. Nucl. Part. Sci. 60, 405 (2010).

[5] L. B. Okun, Zh. Eksp. Teor. Fiz. 83, 892 (1982) [Sov. Phys. JETP 56, 502 (1982)].

[6] P. Galison and A. Manohar, Phys. Lett. 136B, 279 (1984).

[7] B. Holdom, Phys. Lett. 166B, 196 (1986).

[8] S. N. Gninenko and N. V. Krasnikov, Phys. Lett. B 513, 119 (2001).

[9] P. Fayet, Phys. Rev. D 75, 115017 (2007).

[10] M. Pospelov, Phys. Rev. D 80, 095002 (2009).

[11] R. Essig et al., arXiv:1311.0029.

[12] J. Alexander et al., arXiv:1608.08632.

[13] M. Battaglieri et al., arXiv:1707.04591.

[14] J. Beacham et al., arXiv:1901.09966.
[15] R. Alemany et al., arXiv:1902.00260.

[16] A. Berlin, N. Blinov, G. Krnjaic, P. Schuster, and N. Toro, Phys. Rev. D 99, 075001 (2019).

[17] C. Patrignani et al. (Particle Data Group), Chin. Phys. C 40, 100001 (2016).

[18] S. Abrahamyan et al. (APEX Collaboration), Phys. Rev. Lett. 107, 191804 (2011).

[19] H. Merkel, P. Achenbach, C. Ayerbe Gayoso, T. Beranek, J. Beričič, J. C. Bernauer, R. Böhm, D. Bosnar, L. Correa, and L. Debenjak et al., Phys. Rev. Lett. 112, 221802 (2014).

[20] J. P. Lees et al. (BABAR Collaboration), Phys. Rev. Lett. 113, 201801 (2014).

[21] A. Adare et al. (PHENIX Collaboration), Phys. Rev. C 91, 031901 (2015).

[22] J. R. Batley et al. (NA48/2 Collaboration), Phys. Lett. B 746, 178 (2015).

[23] A. Anastasi et al. (KLOE-2 Collaboration), Phys. Lett. B 757, 356 (2016).

[24] P. deNiverville, M. Pospelov, and A. Ritz, Phys. Rev. D 84, 075020 (2011).

[25] E. Izaguirre, G. Krnjaic, P. Schuster, and N. Toro, Phys. Rev. D 91, 094026 (2015).

[26] E. Izaguirre, G. Krnjaic, P. Schuster, and N. Toro, Phys. Rev. Lett. 115, 251301 (2015).

[27] E. Izaguirre, Y. Kahn, G. Krnjaic, and M. Moschella, Phys. Rev. D 96, 055007 (2017).

[28] L. Darmé, S. Rao, and L. Roszkowski, J. High Energy Phys. 12 (2018) 014; 03 (2018) 084.

[29] E. W. Kolb and M. S. Turner, Front. Phys. 69, 1 (1990).

[30] H. S. Lee, Phys. Rev. D 90, 091702(R) (2014).

[31] M. D. Diamond and P. Schuster, Phys. Rev. Lett. 111, 221803 (2013).

[32] H. Davoudiasl, H. S. Lee, and W. J. Marciano, Phys. Rev. D 89, 095006 (2014).

[33] R. Essig, J. Mardon, M. Papucci, T. Volansky, and Y. M. Zhong, J. High Energy Phys. 11 (2013) 167.

[34] B. Batell, M. Pospelov, and A. Ritz, Phys. Rev. D 80, 095024 (2009).

[35] B. Batell, R. Essig, and Z. Surujon, Phys. Rev. Lett. 113, 171802 (2014).

[36] D. Banerjee, V. Burtsev, D. Cooke, P. Crivelli, E. Depero, A. V. Dermenev, S. V. Donskov, F. Dubinin, R. R. Dusaev, S. Emmenegger et al. (NA64 Collaboration), Phys. Rev. Lett. 118, 011802 (2017).

[37] A. A. Aguilar-Arevalo, M. Backfish, A. Bashyal, B. Batell, B. C. Brown, R. Carr, A. Chatterjee, R. L. Cooper, P. deNiverville, R. Dharmapalan et al. (MiniBooNE Collaboration), Phys. Rev. D 98, 112004 (2018).

[38] D. Banerjee, V. E. Burtsev, A. G. Chumakov, D. Cooke, P. Crivelli, E. Depero, A. V. Dermenev, S. V. Donskov, F. Dubinin, R. R. Dusaev et al. (NA64 Collaboration), Phys. Rev. D 97, 072002 (2018).

[39] J. P. Lees et al. (BABAR Collaboration), Phys. Rev. Lett. 119, 131804 (2017).

[40] E. Cortina Gil et al. (NA62 Collaboration), J. High Energy Phys. 05 (2019) 182.

[41] S. N. Gninenko, Phys. Rev. D 85, 055027 (2012).

[42] S. N. Gninenko, Phys. Lett. B 713, 244 (2012).

[43] S. N. Gninenko, Phys. Rev. D 89, 075008 (2014).

[44] S. Andreas et al., arXiv:1312.3309. 
[45] See, for example, http://sba.web.cern.ch/sba/.

[46] D. Banerjee, P. Crivelli, and A. Rubbia, Adv. High Energy Phys. 2015, 105730 (2015).

[47] E. Depero et al., Nucl. Instrum. Methods Phys. Res., Sect. A 866, 196 (2017).

[48] S. N. Gninenko, N. V. Krasnikov, M. M. Kirsanov, and D. V. Kirpichnikov, Phys. Rev. D 94, 095025 (2016).

[49] S. N. Gninenko, D. V. Kirpichnikov, M. M. Kirsanov, and N. V. Krasnikov, Phys. Lett. B 782, 406 (2018).

[50] S. Agostinelli et al. (GEANT4 Collaboration), Nucl. Instrum. Methods Phys. Res., Sect. A 506, 250 (2003).

[51] J. Allison et al., IEEE Trans. Nucl. Sci. 53, 270 (2006); Phys. Rev. D 80, 075018 (2009).

[52] I. Antcheva et al., Comput. Phys. Commun. 180, 2499 (2009).

[53] E. Gross, LHC statistics for pedestrians, CERN Report No. CERN-2008-001, 2008, p. 71.
[54] T. Junk, Nucl. Instrum. Methods Phys. Res., Sect. A 434, 435 (1999).

[55] G. Cowan, K. Cranmer, E. Gross, and O. Vitells, Eur. Phys. J. C 71, 1554 (2011).

[56] A. L. Read, J. Phys. G 28, 2693 (2002).

[57] H. Davoudiasl and W. J. Marciano, Phys. Rev. D 92, 035008 (2015).

[58] We have made the calculations based on semianalytical formulae of Ref. [29] and found that for pseudo-Dirac (Majorana) fermions $f=0.3-0.4(4.2-5.3)$ for the mass range $1 \leq m_{A^{\prime}} \leq 100 \mathrm{MeV}$. For limit calculations shown in Fig. 4 we used the conservative estimate with $f=0.25(3)$ similar to Refs. [13,38].

[59] R. Essig, A. Manalaysay, J. Mardon, P. Sorensen, and T. Volansky, Phys. Rev. Lett. 109, 021301 (2012). 